Pontifícia Universidade $_{\text {do Rio de Janeiro }}$

Aellington Freire de Araújo

\title{
Redistribuição de momentos em vigas contínuas em aduelas protendidas com cabos sintéticos externos
}

Tese de Doutorado

Tese apresentada como requisito parcial para obtenção do grau de Doutor pelo Programa de Pós-Graduação em Engenharia Civil da PUC-Rio. Área de Concentração: Estruturas.

Orientador: Giuseppe Barbosa Guimarães

Rio de Janeiro, 27 Fevereiro de 2003 
Pontifícia Universidade Católica

DO RIO DE JANEIRO

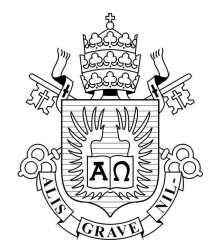

Aellington Freire de Araújo

\title{
Redistribuição de momentos em vigas contínuas em aduelas protendidas com cabos sintéticos externos
}

\begin{abstract}
Tese apresentada como requisito parcial para obtenção do grau de Doutor pelo Programa de Pós-Graduação em Engenharia Civil do Departamento de Engenharia Civil do Centro Técnico Científico da PUC-Rio. Aprovada pela Comissão Examinadora abaixo assinada.
\end{abstract}

Prof. Giuseppe Barbosa Guimarães Orientador

Departamento de Engenharia Civil - PUC-Rio

Profa. Claudia Maria Oliveira Campos IME

Profa Deane de Mesquita Roehl Departamento de Engenharia Civil - PUC-Rio

Prof. Guilherme Sales S. de Azevedo Melo UnB

Prof. Ibrahim Abd El Malik Shehata UFRJ

Prof. Sebastião Artur L. de Andrade Departamento de Engenharia Civil - PUC-Rio

Ney Augusto Dumont

Coordenador Setorial do Centro Técnico Científico - PUC-Rio

Rio de Janeiro, 27 de Fevereiro de 2003 
Todos os direitos reservados. É proibida a reprodução total ou parcial do trabalho sem autorização da universidade, do autor e do orientador.

\section{Aellington Freire de Araújo}

Graduou-se em Engenharia Civil pela na UFPB (Universidade Federal da Paraíba). Na UFPB, participou de Iniciação Científica na área de Materiais nãoconvencionais. Na PUC-Rio desenvolveu seu trabalho de Mestrado e Doutorado com ênfase em Análise Experimental de Estruturas.

Ficha Catalográfica

Araújo, Aellington Freire de

Redistribuição de momentos em vigas contínuas em aduelas protendidas com cabos sintéticos externos / Aellington Freire de Araújo; orientador: Giuseppe Barbosa Guimarães. - Rio de Janeiro : PUC, 2003.

[14], 255 f. ; $30 \mathrm{~cm}$

Tese (doutorado) - Pontifícia Universidade Católica do Rio de Janeiro, Departamento de Engenharia Civil.

Inclui referências bibliográficas.

1. Engenharia civil - Teses. 2. Redistribuição de momentos. 3. Concreto protendido. 4. Protensão externa. 5. Cabo sintético. 6. Aduela prémoldada. I. Guimarães, Giuseppe Barbosa. II. Pontifícia Universidade Católica do Rio de Janeiro. Departamento de Engenharia Civil. III. Título. 
“...No caminho de nossa felicidade encontraremos o conhecimento para o qual escolhemos esta vida... “

\section{Richard Bach}

\section{Ilusões}

A toda a minha família, principalmente a minha mãe Iône, a minha irmã Celly, aos meus sobrinhos Wanderson e Wellyson, a minha tia Irêne, aos meus avós: seu Irineu (em alma) e a dona Maria. 


\section{AGRADECIMENTOS}

Ao professor Giuseppe Barbosa Guimarães pela orientação e pela amizade demonstrada ao longo deste trabalho.

\section{Aos Amigos !!!!!!!!!!!!!!}

Ao CNPq pelo auxílio financeiro e a Linear Composites Ltda (Inglaterra) pela doação dos cabos. 


\section{Resumo}

Araújo, Aellington Freire de; Guimarães, Giuseppe Barbosa Redistribuição de momentos em vigas contínuas em aduelas protendidas com cabos sintéticos externos. Rio de Janeiro, 2003. 255p. Tese de Doutorado - Departamento de Engenharia Civil, Pontifícia Universidade Católica do Rio de Janeiro.

Este trabalho apresenta um estudo experimental sobre a redistribuição de momentos em vigas contínuas de concreto construídas em aduelas pré-moldadas, com juntas secas, e protendidas com cabos sintéticos externos. Para tanto foram ensaiadas quatro vigas contínuas, com dois vãos iguais, sendo uma monolítica e as demais em aduelas pré-moldadas. Todas as vigas têm a mesma seção transversal tipo I, com dois vãos, altura da seção de $30 \mathrm{~cm}$, largura do flange e espessura da alma igual a $30 \mathrm{~cm}$ e $10 \mathrm{~cm}$, respectivamente. A viga monolítica tem relação $l / d_{p}$ (vão/altura) igual a 18,75 e nas vigas em aduelas os valores desta relação foram entre $12,5,18,75$ e 25 . As vigas são protendidas com dois cabos sintéticos externos com traçado poligonal. Os cabos utilizados são feitos com fibras sintéticas de alta resistências (2700 MPa) e alto modulo de elasticidade (126000 MPa) conhecidas comercialmente como Kevlar. O objetivo deste estudo é examinar a redistribuição de momentos em vigas contínuas com sistemas construtivos diferentes e com diferentes relações $1 / \mathrm{d}_{\mathrm{p}}$ frente à utilização de cabos sintéticos. Os resultados mostram que a redistribuição de momentos em vigas em aduelas pode ser obtida a partir de uma viga monolítica semelhante e que o comportamento da redistribuição de momentos nas vigas em aduelas é pouco influenciada pela relação $1 / d_{p}$.

\section{Palavras-chave}

Redistribuição de momentos; concreto protendido; cabo externo; cabo sintético; aduela pré-moldada. 


\section{Abstract}

Araújo, Aellington Freire de; Guimarães, Giuseppe Barbosa. Moment redistribution in continuous segmental beams prestressed with external synthetic tendons. Rio de Janeiro, 2003. 255p. D.Sc. Thesis Department of Civil Engineering, Pontifícia Universidade Católica do Rio de Janeiro.

An experimental investigation on the behavior of continuous segmental concrete beams, prestressed with external aramid tendons, was carried out. Four beams were tested; one was monolithic and the others were constructed in precast dry-jointed segments. All the beams had the same overall dimensions, with two spans and an I section $30 \mathrm{~cm}$ high, flange width of $30 \mathrm{~cm}$ and web thickness of $10 \mathrm{~cm}$. The $l / d_{p}$ (span/section height) ratio was 18,75 for the monolithic beam and 12.5, 18.75 and 25 for the segmental beams. The beams were post-tensioned with two external aramid tendons, which are made of high strength (2700 MPa) high modulus (126000 MPa) Kevlar 49 yarns. The main objective was to study the moment redistribution in the post-cracking stage. Test results have shown that the moment redistribution in segmental beams can be obtained from a similar monolithic beam and that the moment redistribution is only slightly affected by the $l / d_{p}$.

\section{Keywords}

Moment redistribution; concrete prestressed; external tendon; synthetic tendon; precast segment. 


\section{Sumário}

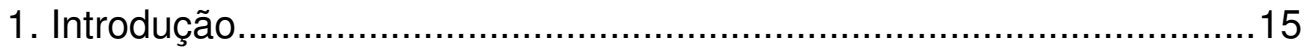

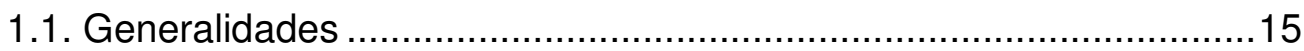

1.2. Vigas construídas a partir de aduelas pré-moldadas........................16

1.3. Protensão externa com cabos sintéticos ...........................................17

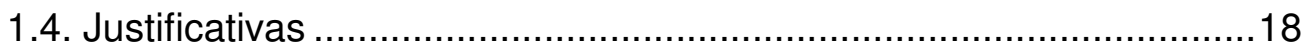

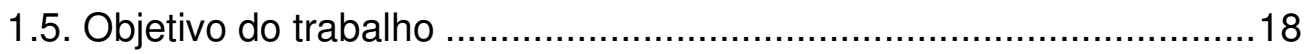

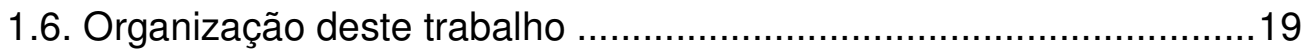

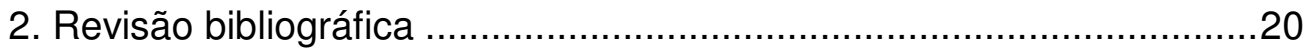

2.1. Introdução

2.2 Comportamento de vigas construídas a partir de aduelas

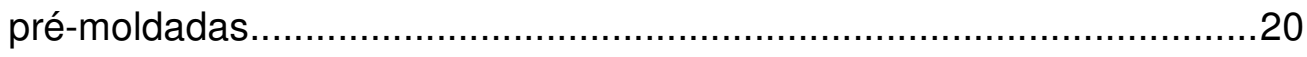

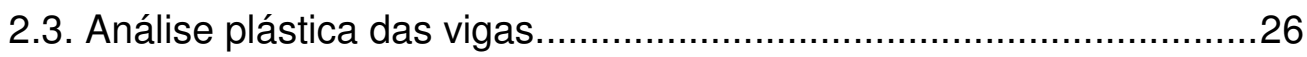

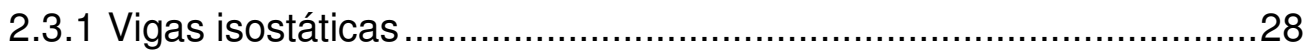

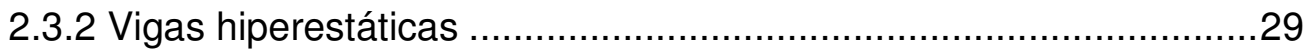

2.4. Capacidade de rotação da rótula plástica .........................................39

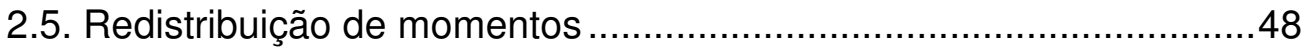

2.5.1 Vigas protendidas com cabos de aço .......................................... 48

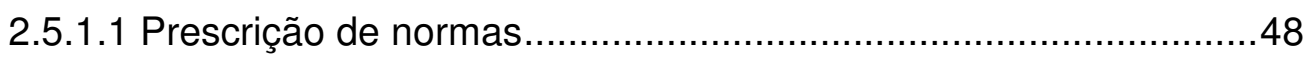

2.5.1.2 Consideração segundo diferentes estudos ................................52

2.5.2 Vigas com cabos sintéticos ……………………........................

3. Ensaios preliminares sobre concentração de deformações

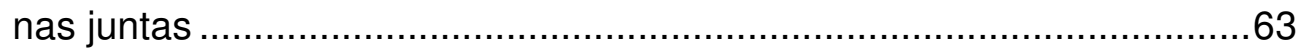

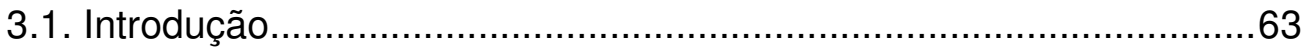

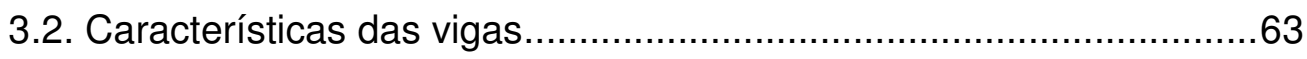

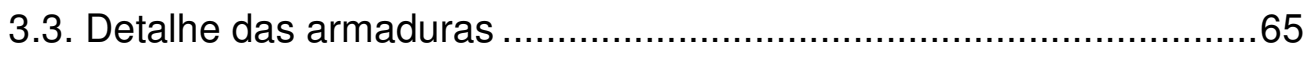

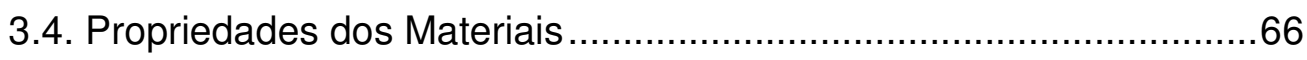

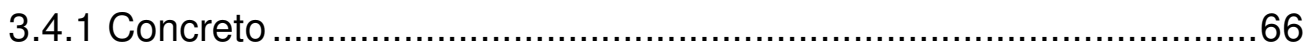

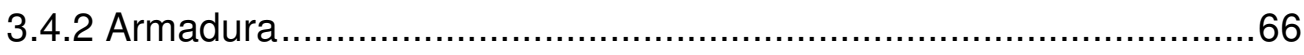

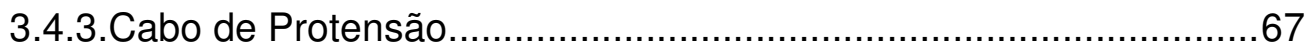


3.5. Montagem dos ensaios e Instrumentação.........................................68

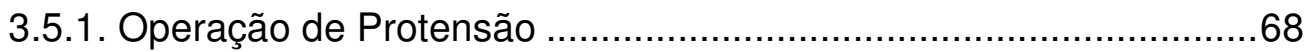

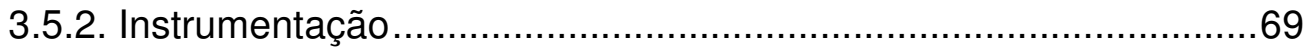

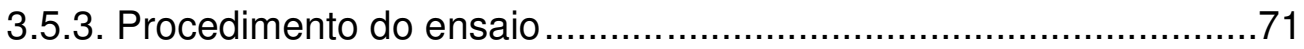

3.6. Apresentação e discussão dos resultados …………………..........72

3.6.1. Apresentação dos resultados. ..................................................

3.6.1.1. Deslocamento, deformação, força nos cabos e abertura das

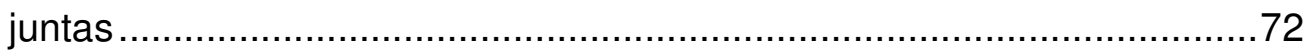

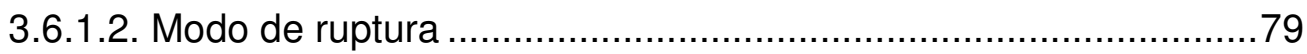

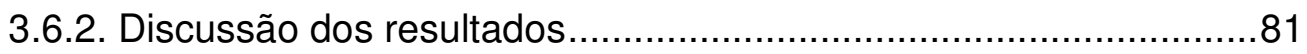

3.6.2.1. Momento atuante vs. Abertura da junta .................................... 81

3.6.2.2. Momento atuante vs. Deformação ......................................... 82

3.6.2.3. Variação da Força nos cabos de protensão ................................. 86

3.6.2.4. Efeito da concentração de deformação no

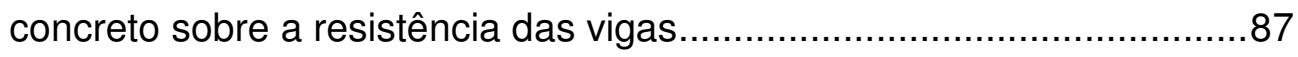

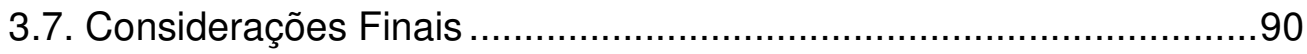

4. Ensaios principais: descrição e apresentação dos resultados ..............91

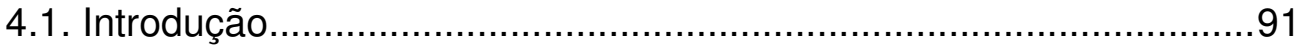

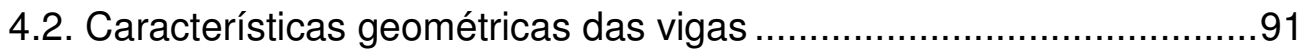

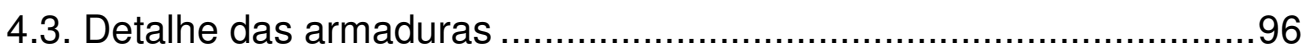

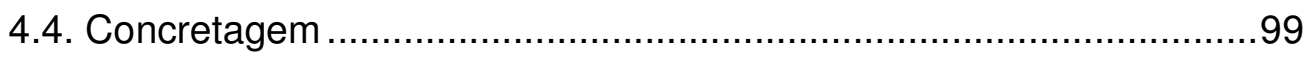

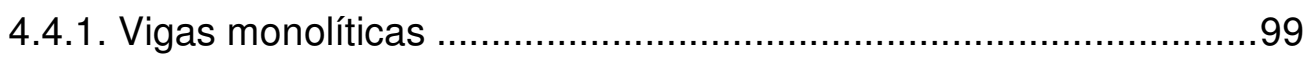

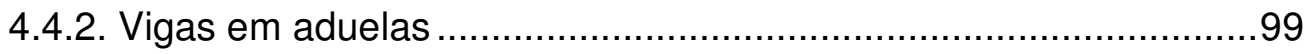

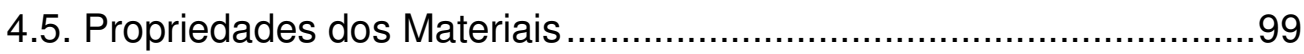

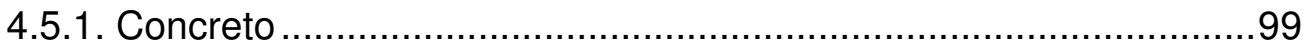

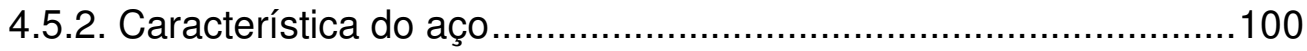

4.5.3. Características dos cabos de protensão ……………………....102

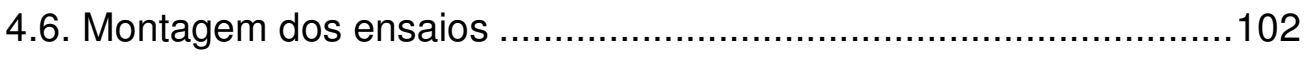

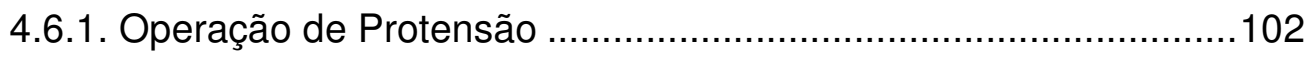

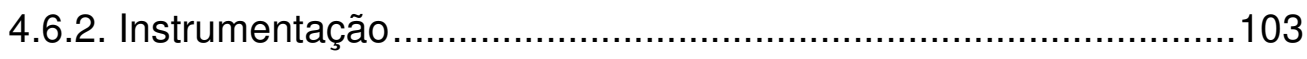

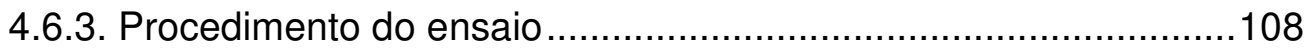

4.7. Apresentação dos resultados ....................................................110 


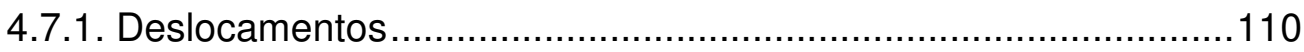

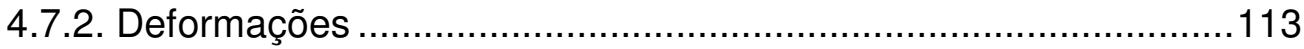

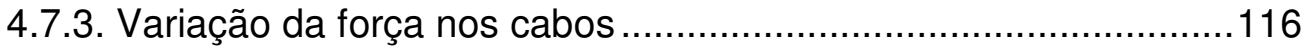

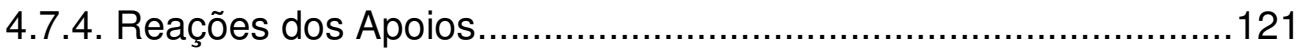

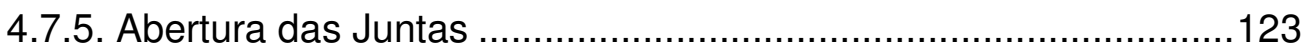

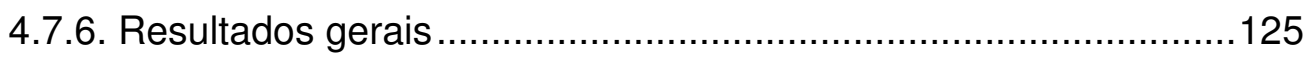

5. Ensaios principais: análise dos resultados .....................................129

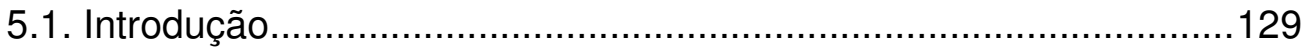

5.2. Vigas monolítica e em aduelas, com mesma relação $\mathrm{l} / \mathrm{d}_{\mathrm{p}}=18,75 \ldots 129$

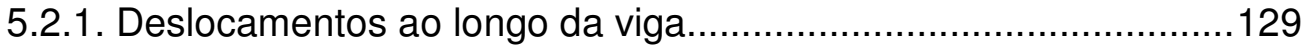

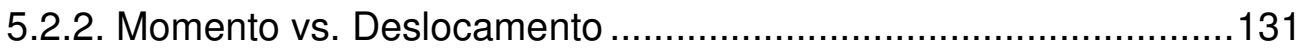

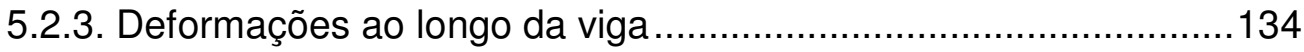

5.2.4 Variação da força nos cabos de protensão ..................................138

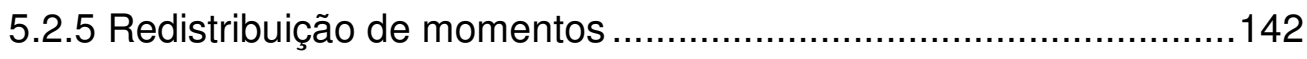

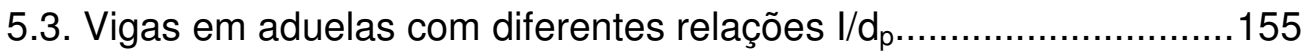

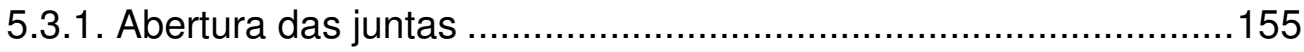

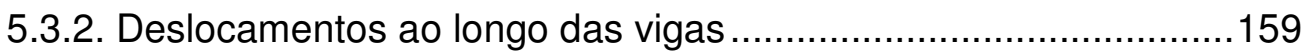

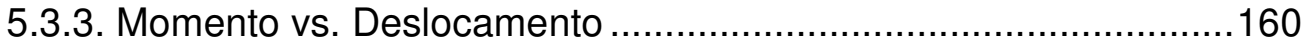

5.3.4. Deformação no concreto ao longo da viga ..................................162

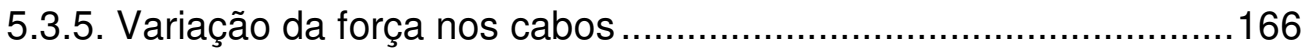

5.3.6 Redistribuição de momentos nas vigas em aduelas com

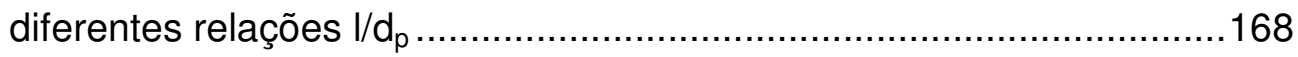

5.5. Comparação entre os resultados experimentais e teóricos.............175

6. Conclusões e sugestões para trabalhos futuros................................181

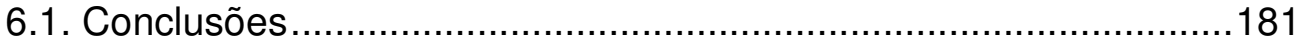

6.2. Sugestões para trabalhos futuros...............................................185

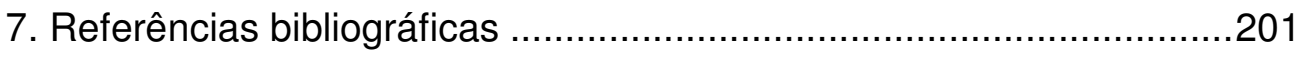




\section{Lista de símbolos}

\section{ROMANOS}

$A_{p} \quad$ - área de armadura protendida

$A_{s} \quad$ - área de armadura passiva (tração)

$A_{\text {'s }} \quad$ - área de armadura passiva (compressão)

b - largura da seção

$b_{f} \quad$ - largura da mesa

$b_{w} \quad$ - largura da alma

d - distância da fibra comprimida até o centróide da armadura passiva de tração

$d_{p} \quad$ - distância da fibra comprimida até o centróide da armadura de protensão

$d_{s} \quad$ - altura da fibra mais comprimida ao centróide da armadura passiva de tração

e - excentricidade do cabo

$\mathrm{El}_{\mathrm{C}}$ - rigidez à flexão na ruptura na seção crítica do apoio

$\mathrm{El}_{\mathrm{Cy}}$ - rigidez à flexão da seção no apoio no início do escoamento da armadura passiva

$E_{s} \quad$ - módulo de elasticidade da armadura de passiva

$\mathrm{E}_{\mathrm{ps}} \quad$ - módulo de elasticidade da armadura de protensão

$f^{\prime} c$ - resistência à compressão do concreto

$\mathrm{f}_{\mathrm{ck}}$ - resistência à compressão característica do concreto

$\mathrm{f}_{\mathrm{cm}}$ - resistência média de compressão dos corpos de prova de concreto

$f_{p e} \quad$ - tensão efetiva da armadura de protensão

$f_{\mathrm{ps}} \quad$ - tensão última da armadura de protensão

$f_{\mathrm{pu}} \quad$ - resistência última da armadura de protensão

$f_{\mathrm{py}} \quad$ - tensão de escoamento do aço de protensão

$f_{y} \quad$ - tensão de escoamento da armadura passiva de tração

$f_{y}^{\prime} \quad$ - tensão de escoamento da armadura passiva compressão

$\mathrm{F}_{\mathrm{a}}$ - força nos cabos de protensão medidas nas ancoragem ativa

$F_{p} \quad$ - força nos cabos de protensão medidas nas ancoragem passiva

$F_{\circ} \quad$ - força de protensão inicial 


$$
\begin{aligned}
& \mathrm{F}_{3} \quad \text { - força de protensão inicial do ciclo } 3 \\
& \mathrm{~F}_{4} \quad \text { - força de protensão inicial do ciclo } 4 \\
& F_{r} \quad \text { - força de protensão na ruptura } \\
& h_{f} \text { - espessura do flange } \\
& \text { h - altura da seção } \\
& \text { K - fator de atrito } \\
& I_{p} \quad \text { - comprimento de rótula plástica } \\
& \text { I - comprimento do vão } \\
& \text { L - comprimento do vão } \\
& \mathrm{Ma}_{\mathrm{el}} \quad \text { - momento elástico no apoio central } \\
& \mathrm{Ma}_{\mathrm{exp}} \quad \text { - momento experimental no apoio central } \\
& M_{\text {apoio }} \quad \text { - momento resistente no apoio. } \\
& M_{c r} \quad \text { - momento de fissuração } \\
& M_{d, \max } \quad \text { - momento fletor desta seção que está mais solicitada à flexão } \\
& \mathrm{M}_{\mathrm{e}} \quad \text { - momento de escoamento } \\
& M_{\max } \quad \text { - momento máximo aplicado em uma seção } \\
& M_{p} \quad \text { - momento de plastificação } \\
& M_{\mathrm{pc}} \quad \text { - momento último no apoio central } \\
& \mathrm{Mp}_{\mathrm{el}} \quad \text { - momento elástico no ponto de aplicação de carga } \\
& \mathrm{Mp}_{\mathrm{exp}} \quad \text { - momento experimental no ponto de aplicação de carga } \\
& M_{p s} \quad \text { - momento último na seção critica do vão } \\
& M_{R} \quad \text { - momento resultante } \\
& M_{\text {sec }} \quad \text { - momento secundário } \\
& \mathrm{M}_{\mathrm{u}} \quad \text { - momento último } \\
& M_{\text {vão }} \quad \text { - momento de resistente do vão } \\
& M_{0} \quad \text { - momento de descompressão numa seção } \\
& \mathrm{M}_{1} \quad \text { - momento primário } \\
& \mathrm{M}_{2} \quad \text { - momento secundário } \\
& \text { n - número total de vãos } \\
& \text { P - carga concentrada aplicada } \\
& P_{e} \quad \text { - carga concentrada de escoamento } \\
& \mathrm{P}_{\mathrm{r}} \quad \text { - carga concentrada de ruptura } \\
& \mathrm{P}_{\mathrm{u}} \quad \text { - carga concentrada última } \\
& P_{\text {col }} \quad \text { - capacidade de carga última }
\end{aligned}
$$


$\mathrm{P}_{\mathrm{el}} \quad$ - capacidade de carga baseada na teoria elástica - linear

$\mathrm{P}_{\mathrm{pl}} \quad$ - capacidade de carga obtida na análise plástica

PAR - relação de adaptação plástica

$R_{c} \quad$ - força resultante no concreto

$R_{p}$ - força de protensão

$R_{S} \quad$ - força na armadura passiva de tração

$\mathrm{V}_{\mathrm{a}}$ - força devido ao efeito do engrenamento do agregado

$\mathrm{V}_{\mathrm{c}}$ - forças oriundas de mecanismos complementares ao modelo em treliça

$\mathrm{V}_{\mathrm{cv}} \quad$ - componente vertical da força resultante de compressão

$V_{d} \quad$ - força devido ao efeito de pino

$V_{s}$ - resultante das forças que atuam na armadura transversal

$\mathrm{V}_{\mathrm{u}} \quad$ - força cortante última

$x \quad$ - profundidade da linha neutra

z $\quad$ - braça de alavanca entre a força resultante de compressão e a força de protensão

$\mathrm{Z}_{\mathrm{s}} \quad$ - braço de alavanca em relação ao cabo de protensão

$\mathrm{Z}_{\mathrm{s}} \quad$ - braço de alavanca em relação à armadura passiva

\section{GREGOS}

$\kappa_{\mathrm{e}} \quad$ - curvatura referente ao escoamento da armadura passiva

$\kappa_{\text {máx }} \quad$ - curvatura máxima

$\eta \quad$ - grau de redistribuição de momentos

$\rho_{\mathrm{s}} \quad$ - taxa de armadura passiva de compressão

$\rho_{p} \quad$ - taxa geométrica de armadura passiva de protensão;

$\rho$ - taxa geométrica de armadura passiva de tração;

$\rho^{\prime} \quad$ - taxa geométrica de armadura passiva de compressão;

$\varepsilon_{\mathrm{uk}} \quad$ - deformação última

$\delta \quad$ - fator de redistribuição

$\omega$ - taxa mecânica de armadura passiva de tração

$\omega^{\prime} \quad$ - taxa mecânica de armadura passiva de compressão

$\omega_{p} \quad$ - taxa mecânica de armadura protendida. 

$\omega_{\mathrm{t}} \quad$ - índice total de armadura
$\phi \quad$ - acréscimo deste ângulo devido aos deslocamentos da viga no ponto de desvio, após a aplicação do carregamento externo.
$\mu \quad$ - coeficiente de atrito
$\theta$ - ângulo desvio do cabo antes da aplicação do carregamento externo 